\title{
Nuevas tendencias para la optimización de los procesos de Inteligencia de Negocios
}

\section{New trends for the optimization of Business Intelligence processes}

\author{
Rosa Mirelly García Jara ${ }^{1}$, Katty Susana Gutiérrez Villanueva ${ }^{2}$, Katherin Vanessa Rodríguez \\ Zevallos $^{3}$, y Frank Edmundo Escobedo Bailón ${ }^{4}$
}

${ }^{1}$ Universidad Nacional Mayor de San Marcos. Av. Universitaria cruce con Av. Venezuela cdra. 34, Lima, Perú. Correo electrónico: rosa.garcia11@unmsm.edu.pe. ORCID: https://orcid.org/0000-0002-2703-6885

${ }^{2}$ Universidad Nacional Mayor de San Marcos. Av. Universitaria cruce con Av. Venezuela cdra. 34, Lima, Perú. Correo electrónico: katty.gutierrez1@unmsm.edu.pe. ORCID: https://orcid.org/0000-0001-7766-7848

${ }^{3}$ Universidad San Ignacio de Loyola. Pasaje Ayancocha 143 - Huánuco, Huánuco, Perú. Correo electrónico: vane251089@hotmail.com. ORCID: https://orcid.org/0000-0001-8350-720X

${ }^{4}$ Universidad Nacional Tecnológica de Lima Sur. Calle Carlos Alayza y Roel 2392 Dpto. 104 - Lince, Lima, Perú. Correo electrónico: fescobedo@untels.edu.pe. ORCID: https://orcid.org/0000-0002-2058-0976

\section{Resumen}

Cada vez la Inteligencia de Negocios se hace más relevante en las empresas, esto se debe a que los tomadores de decisiones se apoyan en ella para realizar la labor propiamente dicha. En base a esta aserción, es necesario el facilitar el proceso de BI, reduciendo los tiempos de respuesta y aumentando la eficacia y eficiencia. En este artículo se muestran diversas maneras de realizar Inteligencia de Negocios, desde el origen, es decir la extracción de los datos, hasta el último eslabón del proceso relacionado con la toma de decisiones. En tal medida, se presenta alternativas novedosas que con el estudio necesario han demostrado que van más allá de lo que ahora conocemos como BI, permitiendo, no solo realizar la toma de decisiones sino planteando que estas tengan un apoyo automatizado, asimismo, permitir que los datos se procesen prácticamente solos y manejar reportes más reales basándonos en datos de diversas fuentes. El objetivo del estudio es analizar las nuevas tendencias para el desarrollo de los procesos relacionados a la Inteligencia de negocios, para lo cual se ha realizado una meticulosa revisión bibliográfica por medio de la consulta de artículos científicos, libros y conferencias científicas. En primer lugar, se ha desarrollado la descripción de términos, y puesta en escena de la información recaudada por medio de la investigación, dedicado a las diversas tendencias innovadoras para el despliegue de la Inteligencia de Negocios, mostrando nuevas definiciones, arquitecturas y tendencias que se están llevando a cabo en la actualidad. Finalmente, las propuestas de arquitectura SOA, adquisición de datos abiertos, automatización de procesos y reingeniería de almacenes de datos permitirían la optimización de la inteligencia empresarial por medio de sus alternativas.

Palabras clave: Inteligencia de Negocios, Proceso ETC, Arquitectura Orientada a Servicios, Almacén de Datos, Inteligencia Computacional.

\begin{abstract}
Business Intelligence is becoming more and more relevant in companies, this is due to the fact that decision makers rely on it to perform the work itself. Based on this assertion, it is necessary to facilitate the BI process, reducing response times and increasing effectiveness and efficiency. This article shows different ways to perform Business Intelligence, from the origin, i.e. the extraction of data, to the last link of the process related to decision making. To this extent, new alternatives are presented that with the necessary study have shown that they go beyond what we now know as BI, allowing not only to make decisions but also to propose that these have an automated support, also allowing the data to be processed practically alone and to handle more real reports based on data from various sources. The objective of the study is to analyze the new trends for the development of processes related to business intelligence, for which a meticulous bibliographic review has been carried out by consulting scientific
\end{abstract}


UNIVERSIDAD NACIONAL DE UCAYALI

Revista de Investigación Universitaria

Versión electrónica $2664-8423$

ARTICULO DE REVISIÓN
Vol. $11 \mathrm{~N}^{\circ} 1$, pp. 524 - 539, enero/junio 2021

Recibido 21/02/2021

Aceptado 16/06/2021

Publicado 30/06/2021

articles, books and scientific conferences. First, the description of terms and the staging of the information collected through the research, dedicated to the various innovative trends for the deployment of Business Intelligence, showing new definitions, architectures and trends that are currently being carried out, have been developed. Finally, SOA architecture proposals, open data acquisition, process automation and data warehouse reengineering would allow the optimization of business intelligence through their alternatives.

Keywords: Business Intelligence, ETL Process, Service Oriented Architecture, Data Warehouse, Computational Intelligence.

\section{Introducción}

Actualmente, las empresas al verse inmersas en los nuevos requerimientos del mercado, donde se tiene que la mayor predisposición se orienta al lado de la tecnología, crece la necesidad de poder adecuarse y utilizar las nuevas alternativas en favor del crecimiento de las utilidades, agilización de los procesos y eficiencia y efectividad de los resultados.

Concerniente a estas tecnologías tenemos a la Inteligencia de Negocios - BI, definida por The Data Warehousing Institute, como la serie de procesos, tecnologías y herramientas necesarias para transformar los datos en información, la información en conocimiento y este último en planes que logren que una decisión de negocios sea rentable (Loshin, 2003). La inteligencia de negocios posee una serie de pasos y una arquitectura tradicional y funcional, con muchos resultados positivos tras su implementación.

En este artículo, se plantean nuevas manera de realizar los procedimientos de $\mathrm{BI}$, para lo cual se tiene, en primer lugar, la agilización del proceso ETL mediante la orientación a servicios, seguido de la automatización del análisis de resultados aplicando inteligencia computacional, la modelización de la arquitectura de BI por medio de la Arquitectura Orientada a Servicios, la optimización del tiempo de procesamiento de solicitudes en el Data Warehouse y, por último, la integración y utilización de los datos abiertos para el análisis comparativo.

Todos estos puntos permitirán ampliar la visión que se tiene de la Inteligencia de Negocios y las oportunidades que significan estas nuevas alternativas.

\section{Antecedentes}

Liyang et al. (2011) “A Conceptual Framework for Business Intelligence as a Service (SaaS BI)". Esta investigación ha permitido visualizar una nueva alternativa para el entorno de de despliegue de las soluciones de Inteligencia de Negocios, con el fin de poder migrar de la tradicional infraestructura de BI, en donde el trabajo muchas veces demanda más recursos de los que las empresas tienen, haciéndolo inmanejable, arriesgado y limitante económicamente. Con la aparición de la computación en la nube y el software como servicio (SaaS), las soluciones de BI se vuelven 
UNIVERSIDAD NACIONAL DE UCAYALI

Revista de Investigación Universitaria

Versión electrónica $2664-8423$

ARTICULO DE REVISIÓN
Vol. $11 \mathrm{~N}^{\circ} 1$, pp. 524 - 539, enero/junio 2021

Recibido 21/02/2021

Aceptado 16/06/2021

Publicado 30/06/2021 cada vez más posibles para empresas incipientes y con mayor manojo de la información y el conocimiento para las grandes empresas.

Mediante el uso de Internet los usuarios de BI ya no requerirían de manejar infraestructuras costosas debido a que todo se encontraría en la red. Esta propuesta de Saas - BI cuenta con 5 capas unificadas, en donde cada componente se conecta con otro como si fuera un servicio, garantizando la disponibilidad y la eficacia de la información que será presentada por la solución, además el coste marginal se reduciría, tendiendo a cero. Otro punto importante es que este tipos de sistemas integrarían no solo los datos de la organización, sino que también adjuntaría aquellos que se encuentran dentro de otras aplicaciones SaaS.

SaaS - BI no es solo una solución para las grandes empresas, sino que además permitirían a las medianas y pequeñas empresas ser parte del emergente mundo de la tecnología.

León (2018) "Sistema de Inteligencia de Negocios Móvil en Tiempo Real para apoyar la toma de decisiones en el área de Operaciones Centrales de Servipag". Esta investigación ayudó a dar solución a un problema en la empresa Servipag, la cual se dedica a rendir información sobre pagos y recaudaciones de servicios, tanto a bancos como a empresas en línea. La dificultad que se presentaba estaba asociada a los reclamos de los clientes por patrones de rendición de cuentas no generados y/o enviados, este problema se daba debido al gran crecimiento a nivel de transacciones que tenía la organización. Por esta razón, se planteó implementar un sistema basado en inteligencia de negocios, que permitiera a los jefes de las áreas de operaciones centrales y rendiciones saber cómo va el proceso de rendición de cuentas en tiempo real. Todo esto a través de la presentación de dashboards en dispositivos celulares, logrando así que los stakeholders estén informados de la situación en todo momento, pudiendo tomar decisiones oportunas y evitando multas por el no cumplimiento de los acuerdos definidos para los envíos.

Este trabajo da un gran referente al inicio del estudio de BI orientado a móviles, además, enumera los múltiples beneficios que tendría el realizar una conexión entre los datos on premise y la nube, entre los cuales está la disponibilidad de la información desde cualquier dispositivo en línea en todo momento. Por otro lado, muestra cómo el campo de la inteligencia de negocios está evolucionando para que pueda adaptarse a las necesidades de cada empresa, y así acelerar los procesos de obtención de datos por parte de los usuarios.

Yulianto, A. (2018) "Implementación de la Inteligencia de Negocios con un enfoque mejorado de toma de decisiones basadas en datos", este estudio tiene como objetivo analizar la creciente importancia que está 
UNIVERSIDAD NACIONAL DE UCAYALI

Revista de Investigación Universitaria

Versión electrónica $2664-8423$

ARTICULO DE REVISIÓN
Vol. $11 \mathrm{~N}^{\circ} 1$, pp. 524 - 539, enero/junio 2021

Recibido 21/02/2021

Aceptado 16/06/2021

Publicado 30/06/2021 teniendo la Inteligencia de Negocios, mediante

la implementación de un Data Warehouse como el núcleo del sistema de toma de decisiones basado en datos, orientado a acrecentar la tasa de matrícula única de los estudiantes de una universidad estatal de Indonesia. Este almacén será alimentado por bases de datos operacionales que al centralizarse acelerarán el proceso de matrícula, además de fusionar los metadatos en una única clave por medio de una aplicación en línea independientemente implementada. El sistema relacionado a BI proporciona indicadores financieros de la familia de los estudiantes que buscan ser admitidos en la universidad para proporcionar el pago aproximado de la matrícula con respecto a la clase social a la que pertenece, identificada por medio de tres aspectos importantes, la información de los postulantes, el rendimiento del sistema de decisión sobre la matrícula única y por último el resultado obtenido del sistema de decisión.

Esta investigación brinda pasos del proceso de realización de BI teniendo en cuenta la implementación de un almacén de datos el cual integra las bases de datos operacionales con la información necesaria y pertinente, además sugiere algunos softwares de BI, open source, como lo es Pentaho Community Edition el cual facilita la presentación de informes, tablas pivotantes de procesamiento analítico en línea y cuadros de mando.
Vélez (2019) "Sistema de inteligencia de negocio para el análisis de los tratamientos de reducción del colesterol”, esta investigación plantea una solución para ayudar a tomar decisiones correctas al momento de recomendar un tratamiento a los pacientes con colesterol alto, este problema puede ser causado por malos hábitos alimenticios, sedentarismo e incluso puede ser hereditario. Es por ello, que los efectos de los diversos tratamientos son distintos para cada tipo de persona, he aquí la importancia de elegir uno que se adecue a las necesidades del paciente, ayude a reducir el nivel de colesterol y a lidiar con las enfermedades derivadas. El interés principal de este trabajo es diseñar e implementar un sistema de inteligencia de negocios, donde con el análisis de la información de los pacientes se determine la eficacia de los tratamientos, que pueden ser farmacológicos, naturales u homeopáticos.

Vélez (2019), para ello realiza un modelo multidimensional tomando como referencia los datos de los pacientes, sus hábitos, tratamientos e indicadores; además, se efectúa la integración de datos a través del proceso ETL y el diseño del cubo OLAP. Finalmente, se presentan los reportes de información en función a los indicadores de Colesterol LDL, presión sistólica y presión diastólica; los cuales contribuyen a la determinación del tratamiento más eficaz, el más perjudicial y las características de los pacientes más propensos 
UNIVERSIDAD NACIONAL DE UCAYALI

Revista de Investigación Universitaria

Versión electrónica $2664-8423$

ARTICULO DE REVISIÓN
Vol. $11 \mathrm{~N}^{\circ} 1$, pp. 524 - 539, enero/junio 2021

Recibido 21/02/2021

Aceptado 16/06/2021

Publicado 30/06/2021 a sufrir un alza en sus niveles de colesterol.

\section{Metodología}

Con el fin de obtener información relevante para esta investigación se realizó la búsqueda, identificación y selección de documentos bibliográficos procedentes de diversas fuentes, utilizando como palabras claves: Inteligencia de Negocios, Data Warehouse, Orientado a Servicios. Obteniendo del portal de la IEEE un total de 45 artículos relacionados, ubicados temporalmente entre 2005 y 2021, de los cuales se seleccionaron 8 presentados en este artículo. Además, se realizó una búsqueda en internet por medio de "Google" de donde se obtuvo un libro relacionado a la Inteligencia de Negocios y diversas páginas reconocidas con valor académico.

\section{Resultados y discusión}

La Inteligencia de Negocios tiene una estrecha relación con los procesos empresariales debido a que la primera otorga una serie de beneficios a las organizaciones que la implementan.

No todos los datos que obtienen las empresas, tanto de fuentes internas como externas, llegan a ser información, del mismo modo que no toda la información concluye en conocimiento (Ver Fig. 1), por lo que la Inteligencia de Negocios llevada a cabo adecuadamente permite que el conocimiento sea utilizado para mejorar la toma de decisiones.

Loshin (2003), enumera los resultados que se obtiene al llevar a cabo un buen proceso de BI:
Incremento de la rentabilidad: BI ayuda a evaluar el valor de la inversión en proyectos y las expectativas de rentabilidad, además de utilizar este conocimiento para distinguir entre los proyectos rentables y no rentables.

Disminución de los costos: BI ayuda a evaluar los costos de la empresa, ya sea una mejora en la gestión de la logística, reducción de costos operativos o inversiones necesarias para la realización de un proceso.

Mejora de la Gestión de las Relaciones con los clientes: BI se usa para analizar la información relacionada a los clientes, permitiendo mejorar la capacidad de respuesta, descubrir oportunidades de ventas y aumentar la lealtad de los clientes.

Disminución de los riesgos: Aplicar BI a diversos datos permite la disminución de riesgos, los cuales pueden relacionarse a riesgos crediticios, de fiabilidad con clientes y proveedores o a la distribución en la cadena de valor.

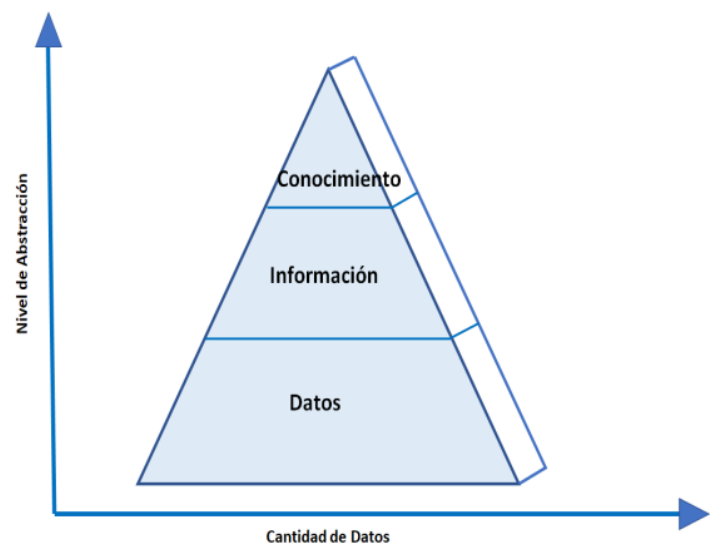

Figura 1: Pirámide de los niveles de abstracción (tomado de Loshin, 2003) 
UNIVERSIDAD NACIONAL DE UCAYALI

Revista de Investigación Universitaria

Versión electrónica $2664-8423$

ARTICULO DE REVISIÓN
Vol. $11 \mathrm{~N}^{\circ} 1$, pp. 524 - 539, enero/junio 2021

Recibido 21/02/2021

Aceptado 16/06/2021

Publicado 30/06/2021
En base a esta información, se han analizado las arquitecturas de las empresas y de la Inteligencia de negocio, con el afán de tener un mejor entendimiento del ambiente en el que ambas variables se desenvuelven.

\section{Arquitectura Empresarial}

En la Fig. 2, se representa la estructura de la Arquitectura Empresarial, la cual está dividida por 5 capas principales, tomando en cuenta la visualización desde la capa base:

- $\quad$ Capa de Infraestructura: en esta se define todo lo relacionado con los componentes físicos del negocio, y su forma de comunicación. Incluyen las plataformas de la arquitectura (dispositivos informáticos, servidores, dispositivos de almacenamiento, etc.), la arquitectura de red, así como los servicios de red requeridos (video, voz, etc.).

- Capa de Datos: aquí se representa el modelo de negocios a través de los esquemas de datos. Se realiza el diseño tanto a nivel conceptual como físico de las bases de datos, data warehouse, repositorios intermedios y demás estructuras que existan dentro de la organización. Además, se definen las herramientas de administración tales como los DBMS, los gestores de contenido, entre otros.

- Capa de Aplicación: este dominio incluye la construcción e implementación de las arquitecturas de los sistemas de información que son necesarios para la realización de los procesos de negocio, así como los que dan apoyo a las actividades de soporte. El desarrollo de estas aplicaciones contribuye a la automatización de las tareas que se realizan y a la gestión de la información de la organización.

- Capa de Negocio: en esta capa se representa de manera conceptual el flujo de los procesos de negocio, los actores que participan, los eventos que podrían ocurrir, así como las diversas tecnologías alineadas a cada actividad. Asimismo, se señala la forma en que estas contribuyen al logro de las estrategias planteadas.

- Capa Estratégica: en la parte superior de la arquitectura se ubica la capa estratégica, en esta se define el marco en el cual se plantean las directrices para el logro de los objetivos, alineándose tanto a la misión como a la visión establecidas por la organización.

En el modelo de arquitectura establecido en la Fig. 2, también se definen dos capas paralelas:

- $\quad$ Capa de Seguridad: conformado por el Sistema de Gestión de la Seguridad de la Información (SGSI), donde se definen los riesgos encontrados en cada proceso de negocio, los controles, las reglas de autenticación y las políticas de seguridad necesarias, para evitar que el impacto generado por la manifestación de la amenaza sea alto. 
UNIVERSIDAD NACIONAL DE UCAYALI

Revista de Investigación Universitaria

Versión electrónica $2664-8423$

ARTICULO DE REVISIÓN
Vol. $11 \mathrm{~N}^{\circ} 1$, pp. 524 - 539, enero/junio 2021

Recibido 21/02/2021

Aceptado 16/06/2021

Publicado 30/06/2021

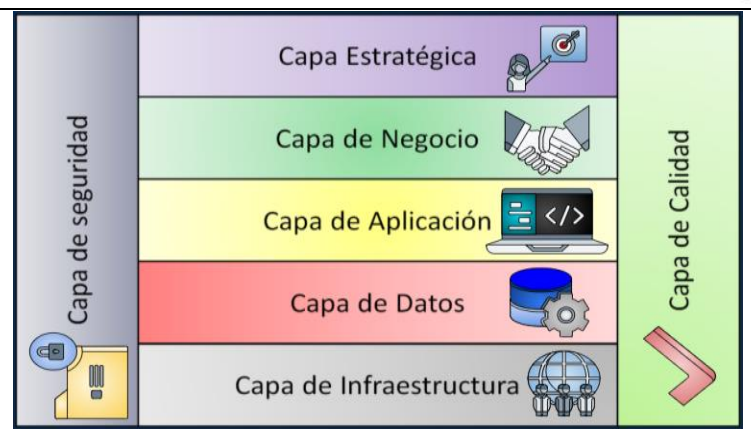

Figura 2: Arquitectura Empresarial (tomado de Hernández-Julio et al., 2017)

- Capa de calidad: delimita los atributos de calidad de cada una de las capas principales siguiendo un estándar como CMMI o la ISO 15504, que ayude a definir el nivel de capacidad o madurez de la organización en el desempeño de sus procesos.

\section{Arquitectura de Inteligencia de Negocios}

En la Fig. 3, se representa la arquitectura de la Inteligencia de Negocios, en ella se puede apreciar, tomando en cuenta la visualización de izquierda a derecha:

- Fuentes de datos: Son todos aquellos contenedores de los cuales se puede extraer los datos, simultáneamente, con la finalidad de ser utilizados en las siguientes etapas de BI. La principal fuente está relacionada con las bases de datos relacionales y no relacionales de la empresa, además de los datos de fuentes externas, estructurados y no estructurados, archivos planos, archivos XML, entre otros.

- $\quad$ Procesos ETL: Este está conformado por la Extracción (E), Transformación (T) y Carga (L). En esta etapa los datos de interés adquiridos de las fuentes de datos son extraídas, debido a que no todos los datos cuentan con el mismo formato requerido por la empresa es necesario que se realice la transformación, en donde se limpian los datos para que posteriormente pasen a ser cargados en donde corresponda para su posterior uso.

- Almacén - Capa Física: La carga de datos realizada en la etapa anterior se hace a este almacén, el cual puede ser un Data Warehouse o los Data Marts departamentales. Este punto podría ser considerado el corazón de la inteligencia de negocios pues a partir de ellos se tomará la información para los análisis posteriores.

- Vista de Negocio: Conformado por los Cubos de información, los cuales se utilizan para la previsualización de la información requerida por la empresa.

- Visualización: Referido a los reportes, dashboards, vistas de la información de manera clara y precisa, destinada a la toma de decisiones inteligentes y a la generación del conocimiento empresarial.

Estas etapas de la arquitectura son llevadas a cabo en todo desarrollo de Inteligencia de Negocios.

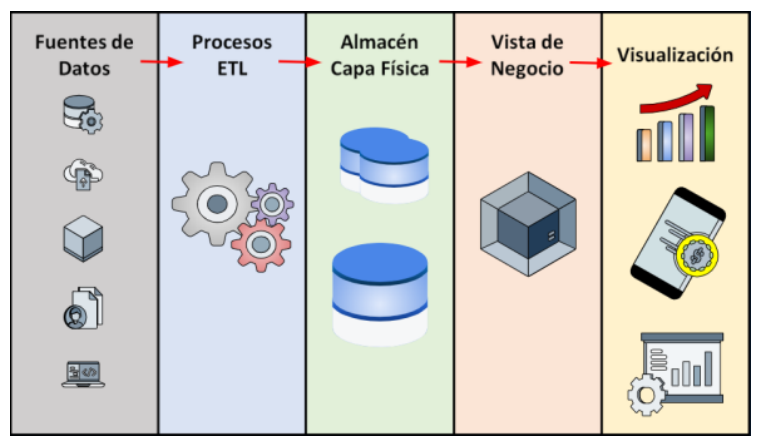

Figura 3: Arquitectura de Inteligencia de Negocios (tomado de Rodríguez \& Bernal, 
UNIVERSIDAD NACIONAL DE UCAYALI

Revista de Investigación Universitaria

Versión electrónica $2664-8423$

ARTICULO DE REVISIÓN
Vol. $11 \mathrm{~N}^{\circ} 1$, pp. 524 - 539, enero/junio 2021

Recibido 21/02/2021

Aceptado 16/06/2021

Publicado 30/06/2021
2019)

Luego de la revisión de diversos documentos se encontraron una serie de temáticas que cada vez están teniendo más acogida cuando se habla de inteligencia de negocios, tanto para la aceleración de procesos de integración, así como para el manejo de grandes volúmenes de datos transaccionales, entre estas tendencias están:

\section{Arquitectura Orientada a Servicios para}

\section{Inteligencia de Negocios}

Desde su origen en los años 60's la Inteligencia de Negocios ha ido escalando y evolucionando hasta llegar a ser lo que hoy conocemos. Todos los aspectos primordiales relatados anteriormente en este artículo se pueden resumir en el objetivo principal de $\mathrm{BI}$ el cual se relaciona con proporcionar una visión procesable e integral del negocio permitiendo que la toma de decisiones sea más viable. Además, se busca tener interpretaciones únicas de los hechos de la organización y contribuir a la gestión del conocimiento empresarial, concepciones que se logran por medio de la arquitectura tradicional. Teniendo en cuenta que cada vez los ambientes de negocios son más dispares los desafíos que afrontan se dificultan considerablemente, entre ellos se encuentran que al trabajar con diferentes fuentes de datos el procesamiento de los mismo se complica haciendo que la presentación de la información pertinente no sea adquirida por quien lo requiere en el momento adecuado, del mismo modo con el acoplamiento de las diversas tecnologías necesarias, las cuales generan redundancia de datos, latencia e imposibilitan el procesamiento en circuito cerrado, lo que quiere decir que la información obtenida al final del proceso de BI no puede ser reutilizada de manera automática por las fuentes de origen. Como solución a tales limitaciones se plantea pasar de la arquitectura tradicional de BI a una Arquitectura Orientada a Objetos.

$\mathrm{Wu}$ et al. (2007), propone una serie de pasos para re - arquitecturizar la construcción de una solución de BI a fin de realizarlo mediante SOA, el cual inicia con la descomposición de los componentes heredados en reutilizables orientados a servicios, con la capacidad de comunicarse por medio de protocolos de mensajería de estándar abierto, basado en XML, Web Services y SOAP.

La parte central de esta solución se encuentra en el almacén de datos (Data Warehouse) el cual contiene información integrada y centralizada, con datos históricos, actuales y predictivos. Este almacén será conformado por un servicio de suscripción en tiempo real y por otros servicios derivados encargados de mantener la coherencia de la información. Estas funcionalidades están relacionadas con el proceso ETL que, en esta propuesta, estará dividido en módulos genéricos de servicios más pequeños, haciéndolo más flexible y escalable y permitiéndole proporcionar datos 
UNIVERSIDAD NACIONAL DE UCAYALI

Revista de Investigación Universitaria

Versión electrónica $2664-8423$

ARTICULO DE REVISIÓN
Vol. $11 \mathrm{~N}^{\circ} 1$, pp. 524 - 539, enero/junio 2021

Recibido 21/02/2021

Aceptado 16/06/2021

Publicado 30/06/2021 significativos, normalizados, bien ensayados y preparados para ser utilizados en distintos proyectos y productos. La integración de una nueva fuente necesitaría solamente de un nuevo modelo de datos XML, lo que reduce significativamente el proceso.

Otro punto importante que es optimizado por medio de SOA es la fase de visualización a través del uso del paradigma editor - suscriptor. Los contenidos del almacén se publican y son accedidos por otros productos mediante interfaces y protocolos de servicios web estándar para que se logre el funcionamiento en cualquier entorno basado en SOA. Asimismo, se puede soportar múltiples herramientas de Inteligencia de Negocios en base a una sola aplicación web que funcione de puente entre todas las herramientas evitando el desarrollo y empaquetamiento de muchas aplicaciones dedicadas a soportar diferentes librerías JDK.

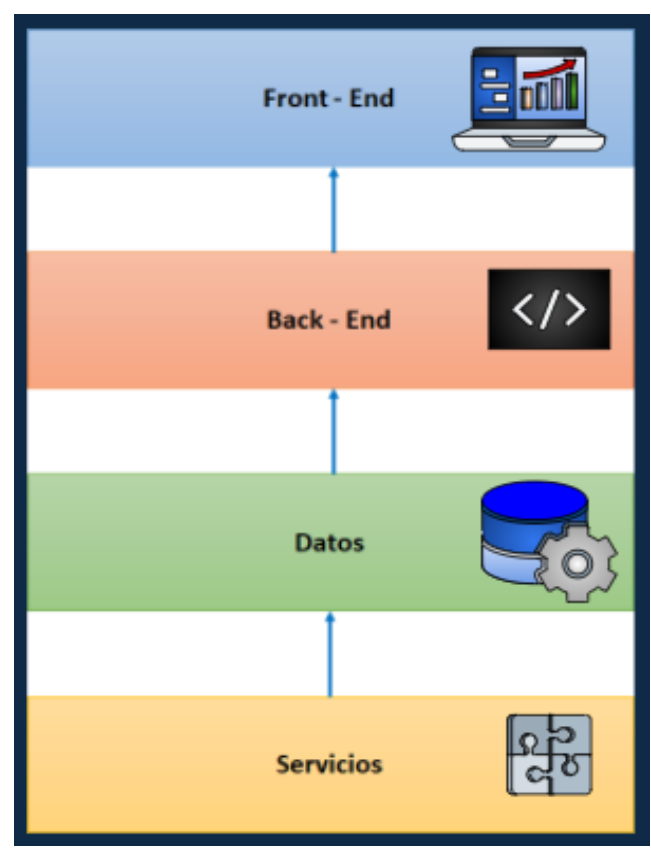

Figura 4: Arquitectura SOA (tomado de Hernández-Julio et al., 2017)

Esta alternativa permite integrar diversas tecnologías en un solo ambiente de inteligencia de negocios, ahorrando costos de estandarización y mantenimiento en entornos evolutivos como los que se presentan actualmente. Pero aunque las ventajas que presenta esta solución son muchas, el principal limitante surge debido a la complejidad del desarrollo de la solución, aun así, con los medios tecnológicos correctos, la viabilidad se potencia.

\section{Inteligencia computacional para inteligencia} de negocios

La Inteligencia Computacional - IC es una metodología para el desarrollo de la computación que posee la capacidad de aprender y afrontar nuevas situaciones, de tal modo que el sistema presenta algunos atributos de razonamiento como la generalización, descubrimiento, asociación y abstracción. (Munóz, 2010)

En base a esta definición se puede decir que las virtudes que presenta la inteligencia computacional servirían de apoyo a la toma de decisiones, poniendo principal atención en las actividades orientadas a la clasificación.

Es así que, Hernández - Julio et al. (2017), propone el desarrollo de un marco basado en la inteligencia computacional aplicado a BI usando SOA, con el objetivo de ayudar en el proceso de tomar decisiones apoyando a la 
UNIVERSIDAD NACIONAL DE UCAYALI

Revista de Investigación Universitaria

Versión electrónica $2664-8423$

ARTICULO DE REVISIÓN
Vol. $11 \mathrm{~N}^{\circ} 1$, pp. 524 - 539, enero/junio 2021

Recibido 21/02/2021

Aceptado 16/06/2021

Publicado 30/06/2021 recolección, pre - información y difusión de la información. Este enfoque toma en cuenta la arquitectura empresarial (Ver Fig. 2) y en base a ello despliega la aplicación de IC principalmente en la capa de aplicación, que en la arquitectura SOA está dividida las capas Back - end y Front - end.

Esta alternativa consta de diversos pasos, pero se extiende principalmente en la preparación de los datos en donde se definen las variables de entrada y de salida para posteriormente ser normalizados y estructurados. Tras el trabajo con la data se debe determinar un predictor y configurar la estructura de la IC, entrenar la técnica establecida para el estudio y finalmente realizar las evaluaciones pertinentes de las medidas definidas antes de que sean visualizados los resultados.

Para la realización del sistema en SOA primero se elige entre las técnicas de inteligencia computacional, los cuales serán implementados por medio de servicios web en donde cada uno encapsula una determinada técnica y por último un servicio encargado de la recomendación basado en los servicios web antes desarrollados.

La inteligencia de negocios se vería mejorada por el despliegue de la inteligencia computacional de tal modo que esta generaría alternativas viables a los decisores teniendo en cuenta decisiones anteriores de las cuales el sistema ha aprendido reduciendo la probabilidad de fallo. Además que por medio de los servicios se lograría agilizar los procesos de visualización para las personas que la requieran en el momento oportuno y con un extra de una decisión prácticamente ya tomada.

\section{$\underline{\text { Inteligencia de Negocios Orientada a Servicios }}$}

La inteligencia de negocios abarca en su desarrollo varios procesos, lograr que estos se realicen de manera eficiente es uno de sus grandes objetivos. Uno de los más importantes y de los que conllevan mayores esfuerzos, es el proceso de integración de datos, en el cual la data proveniente de diversas fuentes pasa por una etapa de transformación y limpieza antes de ser cargada al almacén de datos construido. En general esto suele desarrollarse siguiendo cada una de las fases del proceso ETL, sin embargo, con el desarrollo creciente de las tecnologías ha surgido el concepto de la inteligencia de negocios orientada a servicios (SoBI); esta plantea la utilización de servicios web como un puente entre las fuentes y el almacén de datos, los cuales serán solicitados a través de una tercera aplicación encargada de la gestión de la carga de datos, logrando que la puesta en marcha de la integración se realice con mayor facilidad.

Somya et al. (2018), define el uso de SoBI como una maximización del proceso ETL, que se da con el fin de que las actividades de transformación y carga puedan desarrollarse de manera más sencilla. Asimismo, presenta en su investigación una implementación de SoBI, en la que se busca realizar la integración de los 
UNIVERSIDAD NACIONAL DE UCAYALI

Revista de Investigación Universitaria

Versión electrónica $2664-8423$

ARTICULO DE REVISIÓN
Vol. $11 \mathrm{~N}^{\circ} 1$, pp. 524 - 539, enero/junio 2021

Recibido 21/02/2021

Aceptado 16/06/2021

Publicado 30/06/2021

datos académicos y financieros de la

Universidad Cristiana Satya Wacana haciendo uso de servicios web; para ello se efectúa la construcción de un servicio en cada una de sus aplicaciones transaccionales, los cuales son llamados a través de una aplicación dashboard, para que finalmente el usuario pueda escoger dinámicamente cada uno de los datos que desea almacenar dentro del data warehouse de esquema constelación. Por otro lado, esta solución permite gestionar cada una de las actualizaciones dadas en las bases de datos transaccionales, cargándolas de manera coherente y conveniente en el repositorio centralizado.

A pesar de que el término SoBI aún no es muy común, ya se están presentando varias implementaciones, e irán aumentando a medida que aparezcan nuevas tecnologías y a su vez crezca la necesidad de comunicación entre estas. He aquí que el uso de servicios web orientado a las soluciones de inteligencias de negocios se ubica como una solución a este problema, ya que es capaz de brindar una arquitectura adecuada para soportar la integración de nuevas fuentes de datos sin afectar el proceso de las ya existentes, facilitando la escalabilidad de las aplicaciones BI.

\section{Reingeniería del Almacén de Datos}

El aumento de la cantidad de datos no estructurados que circulan alrededor de los sistemas de información es un aspecto

importante para considerar, ya que estos cada vez son más fundamentales para el logro de los objetivos de las organizaciones. Este crecimiento se da debido al gran número de tecnologías interconectadas que están apareciendo gracias al internet de las cosas. Con esta nueva tendencia surge la pregunta de ¿cómo gestionar los almacenes que contienen toda esta data, para que puedan resolver todas las consultas empresariales requeridas, de manera eficiente?

La dificultad presentada en el personal para el mantenimiento de estos almacenes crece cada día, y más aún con las demandas por parte de los altos ejecutivos, quienes buscan que se reduzca el tiempo de ejecución de las consultas de grandes volúmenes de datos. Como primera proposición, se planteó la optimización de las consultas como posible solución, pero estas no son capaces de predecir los cambios en uso y disposición de los recursos; he ahí que aparece el nuevo paradigma de la reingeniería del almacén de datos, que busca disminuir el tiempo de procesamiento de solicitudes a través del uso eficiente de los recursos.

Sokolov y Turkin (2018), estudia dos métodos importantes para la reingeniería del almacén de datos, el primero de ellos es la indexación, el cual busca evitar el recorrido de toda una tabla al momento de realizar una consulta; y el segundo es la clasificación, método en el cual se priorizan las solicitudes más comunes con el fin de acelerar el proceso. Para ambos, se 
UNIVERSIDAD NACIONAL DE UCAYALI

Revista de Investigación Universitaria

Versión electrónica $2664-8423$

ARTICULO DE REVISIÓN
Vol. $11 \mathrm{~N}^{\circ} 1$, pp. 524 - 539, enero/junio 2021

Recibido 21/02/2021

Aceptado 16/06/2021

Publicado 30/06/2021 introducen una serie de reglas que indican la manera de modificar las claves de distribución y clasificación siguiendo un conjunto de algoritmos que brindarán sugerencias con diversos grados de confianza. Estas permitirán tomar decisiones en base a los pesos dados a las reglas, teniendo como fin la reestructuración de manera óptima del repositorio centralizado. La propuesta que presenta se considera como una buena solución parcial e intermedia al problema de la administración de grandes cantidades de datos, sin embargo, los autores dejan claro que actualmente existen tecnologías modernas que aportan cambios más notables en el rendimiento de los almacenes de datos.

Los esfuerzos por conectarnos a través de diversas tecnologías y obtener datos del ambiente que nos rodea, serán en vano y no nos permitirán tomar decisiones oportunas, si no se encuentra la manera de manejarlos en un entorno adecuado de forma eficiente. Según Reinsel et al. (2018), el volumen de datos para el 2025 será de 175 zettabytes, es por ello que, es de suma importancia profundizar en el estudio de nuevas técnicas que contribuyan a la mejora del desempeño de los almacenes de datos y por ende al desarrollo de la inteligencia de negocios; de manera que, se cumplan las expectativas de los gerentes y los datos capturados sean aprovechados con el fin de obtener ventajas competitivas en la organización.

Uso de Datos abiertos
El término de Datos Abiertos hace referencia a una práctica en la cual cierta data está disponible para todas las personas que lo requieran sin ningún tipo de restricción ni control, esta puede ser distribuida libremente y usada para cualquier fin; un claro ejemplo son tanto las entidades públicas como empresas privadas que han liberado datos para que sean consultados con el objetivo de brindar transparencia en las actividades que realizan. Este nuevo concepto sigue un esquema de datos de cinco estrellas, donde cada estrella define mejores características sobre la forma en que se comparten los datos, teniendo como máximo nivel, cinco estrellas; en este punto los datos pueden ser vinculados con otros, dándoles un contexto para así convertirse en información útil.

Wisnubhadra et al. (2019), implementa una solución que busca apoyar la toma de decisiones sobre la seguridad alimentaria en Indonesia, en este país el desarrollo agrícola es fundamental para el progreso de las aldeas, sin embargo, no se tiene un mecanismo que brinde resultados satisfactorios para la obtención de datos en tiempo real y métricas sobre el rendimiento agrícola. Por esta razón, esta investigación desarrolla un sistema de información de la Aldea y el Área Rural (SIDeKa), que facilita la interacción entre agricultores, proveedores y mercado, así como la adquisición de datos sobre la producción con características de tiempo y espacio. Estos son 
UNIVERSIDAD NACIONAL DE UCAYALI

Revista de Investigación Universitaria

Versión electrónica $2664-8423$

ARTICULO DE REVISIÓN
Vol. $11 \mathrm{~N}^{\circ} 1$, pp. 524 - 539, enero/junio 2021

Recibido 21/02/2021

Aceptado 16/06/2021

Publicado 30/06/2021 integrados en un almacén de datos bajo el concepto de datos abiertos mediante un mecanismo orientado a servicios (SoBI), permitiendo de esta manera responder las preguntas de los usuarios a través del desarrollo de un sistema OLAP.

Se han desarrollado múltiples programas para la entrega de datos abiertos a la población, así como para el uso de estos como una fuente externa de los almacenes de datos en la inteligencia de negocios. Gracias a ello y al nuevo modelo de Internet, la cantidad de datos abiertos crece cada día más, diversificando así sus formas de utilización. Al presentarse esta data con diversos formatos y permitirse el vínculo con otro tipo de datos, se logra que estos sean más dinámicos y brinden información de interés público, que finalmente contribuirán a la toma de decisiones informada en pro de diversos sectores tanto de la población como de la industria.

\section{Casos de éxito en la inteligencia de negocios usando datos abiertos}

La liberación cada vez más notable de datos por parte de los gobiernos, está reforzando el modelo de inteligencia empresarial, contribuyendo así a la transformación de estrategias y operaciones de las organizaciones. Uno de los estudios más contundentes sobre el uso de datos gubernamentales, es el de la empresa Kellogg, multinacional dedicada a la industria agroalimentaria; esta compañía sigue diversos enfoques centrados en el descubrimiento, los cuales permiten identificar tendencias y correlaciones, ayudando de esta manera a satisfacer de mejor manera las necesidades de sus clientes, además de aumentar sus ingresos, con el menor costo. (Rebello et al, 2017)

Rick Davis, vicepresidente de Kellogg, detalla cuatro pasos importantes que esta empresa sigue y que recomienda otras deberían seguir para implementar una estrategia basada en el análisis de datos:

- Formular una pregunta de negocio y una hipótesis que pueda ser probada con el análisis de datos.

- Identificar los conjuntos de datos necesarios (propios y/o gubernamentales)

- Realizar el análisis de los datos para desarrollar conocimientos y probar hipótesis.

- Aplicar los resultados para mejorar su estrategia empresarial.

Otro claro ejemplo de éxito en la utilización de datos abiertos para la aplicación del modelo de inteligencia de negocios, es CVS Health, que desarrolla recomendaciones personalizadas sobre servicios de salud basados en la edad y sexo de las personas, esto con apoyo de los datos gubernamentales del Departamento de Salud y Servicios Humanos de EEUU. Asimismo, Starbucks implementó un sistema BI llamado Atlas, que con ayuda de datos demográficos y meteorológicos del gobierno, logra determinar el impacto de los descuentos 
UNIVERSIDAD NACIONAL DE UCAYALI

Revista de Investigación Universitaria

Versión electrónica $2664-8423$

ARTICULO DE REVISIÓN
Vol. $11 \mathrm{~N}^{\circ} 1$, pp. 524 - 539, enero/junio 2021

Recibido 21/02/2021

Aceptado 16/06/2021

Publicado 30/06/2021 en base al número de usuarios con teléfonos móviles en una determinada zona, así como realizar promociones personalizadas de acuerdo con la temperatura. Best Buy, también utilizó datos demográficos del gobierno para hacer una clasificación de sus clientes en cuatro grupos, de forma que contribuyera a la mejora de la experiencia de compra de sus consumidores tanto en línea como en tienda. (Rebello et al, 2017)

En un futuro se espera que la demanda cada vez mayor de datos abiertos, su liberación por parte del gobierno y las diversas tecnologías emergentes; permitan a las empresas transformar sus modelos de negocios y así aumentar sus ingresos, brindando a su vez mejores productos y servicios a sus clientes. (Rebello et al, 2017)

\section{Direcciones futuras}

La Inteligencia de Negocios ha evolucionado considerablemente y cada año se presentan nuevas alternativas innovadoras, como también la modernización de propuestas realizadas con anterioridad. En estos últimos años el gran auge de los datos y el deseo de las empresas por dominar el sector en el que se desenvuelven son los grandes desencadenantes del cambio.

A medida que las fuentes de datos son más diversas, la gestión de datos en las implementaciones actuales de BI se hace más complejas, por lo que cuanto más personal utiliza datos para impulsar decisiones, las organizaciones deben garantizar la precisión de los mismos (los datos) y su uso en el análisis. (Tableau, 2019)

Según Gartner Inc, para el año 2028 el rendimiento y la escalabilidad recuperarán el protagonismo debido a que la automatización de la información, las tecnologías analíticas y la inteligencia artificial aumentarán la cantidad de datos procesables con el que se cuenta. (Actium Digital, 2020)

Estas predicciones nos permiten apreciar que la gran conversión digital está cada vez más cerca y es importante contar con las herramientas adecuadas para hacerle frente.

El SaaS se ha convertido en el mejor aliado de todos, la computación en la nube ha generado facilidades conllevando a la migración de los datos y aplicaciones, permitiendo el acceso de datos desde cualquier lugar, en cualquier momento (disponibilidad continua). (Qlik, 2021)

Se ha generalizado el uso de datos compartidos, las visualizaciones y la narración de datos, lo que ha dado lugar a que muchas personas hayan tomado interés y hayan iniciado la alfabetización en datos. (Qlik, 2021)

La cantidad de datos con que cuentan las empresas no significan nada si estos no se encuentran actualizados y preparados para responder a los requisitos del negocio, por lo que las alertas, predicciones y actualizaciones se deben realizar con mayor frecuencia, por lo 
UNIVERSIDAD NACIONAL DE UCAYALI

Revista de Investigación Universitaria

Versión electrónica $2664-8423$

ARTICULO DE REVISIÓN
Vol. $11 \mathrm{~N}^{\circ} 1$, pp. 524 - 539, enero/junio 2021

Recibido 21/02/2021

Aceptado 16/06/2021

Publicado 30/06/2021 que se hace necesario contar con procesos más acelerados y con un mejor nivel de respuesta para lo que los sistemas involucrados deben contar con la calidad adecuada. (Qlik, 2021)

Es imprescindible que se capturen y se conecten los datos desde diferentes fuentes alternativas, con el fin de obtener datos derivativos que favorezcan a las nuevas conexiones, asociaciones y combinaciones con los datos propios de la organización. (Qlik, 2021)

\section{Conclusiones}

Si bien es cierto la Inteligencia de Negocios tal como la conocemos ahora es funcional, las nuevas alternativas de desarrollo de la arquitectura basadas en SOA permiten la optimización de los procesos.

La implementación de la inteligencia de negocios orientada a servicios permite que el proceso ETL pierda complejidad debido a que este es realizado de manera más sencilla y sin la intervención de sistemas diversos dedicados al proceso.

La Inteligencia computacional ayuda a que la inteligencia de negocios tenga como resultados en la etapa de visualización alternativas de decisiones más viables.

La utilización de datos abiertos permite integrar fuentes externas que poseen datos relevantes para la inteligencia de negocios en las organizaciones.
Por medio de la reingeniería de los almacenes de datos se aporta la aceleración de los procesos involucrados.

\section{Referencias bibliográficas}

Actium Digital. (2020). 9 tendencias en Business Intelligence. Actium Digital. https://www.actiumdigital.es/es/blog/9tendencias-Business-Intelligence

Hernández-Julio, Y. F., Jimeno, M. A., Leal, N. E., Muñoz, H. y Nieto, W. (2017). Framework for the development of Business intelligence using computational intelligence and serviceoriented architecture. IEEE. 10.1109/ICSESS.2011.5982263

León, A. A. (2018). Sistema de Inteligencia de Negocios Móvil en Tiempo Real para apoyar la toma de decisiones en el área de Operaciones Centrales de Servipag [tesina de maestría, Universidad Técnica Federico Santa María]. Repositorio Institucional MTI.

Liyang, T., Zhiwei, N., Zhangjun, W., \& Li, W. (2011). A Conceptual Framework for Business Intelligence as a Service (SaaS BI). IEEE. 10.1109/icicta.2011.541

Loshin, D. (2003). Business Intelligence (L. Homet, ed.). Morgan Kaufmann Publishers.

Muñoz, J. (2010). Inteligencia Computacional inspirada en la vida. Servicio de Publicaciones de la Universidad de Málaga.

Qlik. (2021). Tendencias de datos y BI para 2021. La gran conversión digital. Qlik. https://www.qlik.com/es-es/bi/datatrends

Rebello, K., Manley, L., Ariss, A. y Gurin, J. (2017). Government Data for Business Innovation in the 21st Century. Accenture.

Reinsel, D., Gantz, J. y Rydning, J. (2018). The Digitization of the World From Edge to Core. IDC. https://resources.moredirect.com/whitepapers/idc-report-the-digitization-ofthe-world-from-edge-to-core

Rodriguez, A. y Bernal, E. (2019). Gestión de 
la información cuantitativa en las universidades. Universidad Nacional de Colombia.

Sokolov, I. y Turkin, I. (2018). Resource Efficient Data Warehouse Optimization. IEEE.

10.1109/DESSERT.2018.8409183

Somya, R., Manongga, D. e Ineke, M. A. (2018). Service-oriented Business Intelligence (SoBI) for Academic and Financial Data Integration in University. IEEE.

10.1109/ISEMANTIC.2018.8549762

Tableau. (2019). Data management converges with modern BI platforms. Tableau. https://www.tableau.com/reports/busine ss-intelligence-trends/data-curation

Vélez, L. T. (2019). Sistema de inteligencia de negocio para el análisis de los tratamientos de reducción del colesterol [tesis de maestría, Universidad Abierta de Cataluña]. Repositorio Institucional UOC.

Wisnubhadra, I., Adithama, S. P. y Kamal S. S. (2019). Agriculture Spatiotemporal Business Intelligence using Open Data Integration. IEEE. 10.1109/ISRITI48646.2019.9034635

Wu, L., Barash, G. y Bartolini, C. (2007). A Service-oriented Architecture for Business Intelligence. IEEE. 10.1109/SOCA.2007.6

Yulianto, A. (2018). Implementation of Business Intelligence With Improved Data-Driven DecisionMaking Approach. IEEE. 10.1109/IIAIAAI.2018.00204 